\title{
Ruthenium-Catalyzed Enyne Cycloisomerizations. Effect of Allylic Silyl Ether on Regioselectivity
}

\author{
Barry M. Trost, Jean-Philippe Surivet and F.Dean Toste \\ Department of Chemistry, Stanford University, \\ Stanford, California, 94305
}

\section{Supplementary Material}

General Techniques. Reactions were carried out in clean, dry glassware under inert atmosphere using high quality solvents. Anhydrous solutions of reaction mixtures were transferred via an oven-dried syringe or cannula. Dichloromethane $\left(\mathrm{CH}_{2} \mathrm{Cl}_{2}\right)$, toluene, acetonitrile and diethyl ether $\left(\mathrm{Et}_{2} \mathrm{O}\right)$ were obtained by passing through commercially available alumina columns. Anhydrous tetrahydrofuran (THF) was obtained by distillation from sodium benzophenone ketyl. Anhydrous acetone was obtained by distillation from freshly activated Drierite). Triethylamine, diisopropylamine, pyridine were distilled from calcium hydride prior to use. Yields refer to chromatographically and spectroscopically homogenous materials. Reagents were purchased at the highest commercial quality and used without further purification. Reactions were monitored by thin layer chromatography separations carried out on 0.2-mm E. Merck silica gel plates (60 F254) using UV light as a visualizing agent and phosphomolybdic acid, $p$-anisaldehyde, or potassium permanganate solutions and heat as developing agents. Flash chromatographies were performed with E. Merck silica gel (0.040-0.063 mm grade). Melting points were obtained on a Thomas-Hoover apparatus in open capillary tubes and are uncorrected. ${ }^{1} \mathrm{H}$ and ${ }^{13} \mathrm{C}$ nuclear magnetic resonance (NMR) were recorded on Varian Gemini-300 (300 and 75MHz respectively) or a Varian Unity Inova 500 (500 and $125 \mathrm{MHz}$ respectively). Chemical shifts are reported in delta $(\delta)$ units, in parts per million ppm) relative to the singlet at $7.24 \mathrm{ppm}$ for chloroform- $d$. Splitting pattern are designated as s, singlet; d, doublet; t, triplet; q, quartet; p, pentet; m, multiplet; and br, broad. Coupling constants $(J)$ are reported in Hertz $(\mathrm{Hz})$. Infrared (IR) data were recorded in sodium plates on PerkinElmer Paragon 500 FT-IR spectrometer. Absorbance frequencies are reported in reciprocal centimeters $\left(\mathrm{cm}^{-1}\right)$. Optical rotation data were obtained with a Jasco DIP-360 digital polarimeter at the sodium D line $(589 \mathrm{~nm})$ in the solvent and concentration indicated. High resolution mass spectral (HRMS) data were obtained from Mass Spectrometry Resource, School of Pharmacy, University of California-San Francisco, on a Kratos MS9 spectrometer. Elemental analyses (Anal.) were performed by M-H-W Laboratories of Phoenix, AZ.

(E)-4,4-(Bismethoxycarbonyl)-1-(tert-butyldimethylsilyl)-oct-7-yn-1-ol (6) 
To a degassed solution of $\left(\eta^{3}-\mathrm{C}_{3} \mathrm{H}_{5} \mathrm{PdCl}\right)_{2}(0.035 \mathrm{~g}, 0.095 \mathrm{mmol})$, triphenylphosphine $(0.201 \mathrm{~g}, 0.76 \mathrm{mmol})$ and cesium carbonate $(1.37 \mathrm{~g}, 4.22 \mathrm{mmol})$ in THF $(15 \mathrm{~mL})$, were added alkyne $\mathbf{5}(0.85 \mathrm{~g}, 5 \mathrm{mmol})$ and methyl carbonate 4 $(1 \mathrm{~g}, 3.84 \mathrm{mmol})$. The reaction mixture was heated at $60^{\circ} \mathrm{C}$ for $2 \mathrm{~h}$, and then partitioned between $\mathrm{Et}_{2} \mathrm{O}(20 \mathrm{~mL})$ and $1 \mathrm{~N} \mathrm{NaHSO}_{4}(20 \mathrm{~mL})$. The aqueous layer was extracted with $\mathrm{Et}_{2} \mathrm{O}(2 \times 20 \mathrm{~mL})$. The combined organic layers were dried over magnesium sulfate $\left(\mathrm{MgSO}_{4}\right)$, and concentrated in vacuo. The residue was chromatographed eluting with 6:1 petroleum ether : diethyl ether to afford enyne $\mathbf{6}(0.840 \mathrm{~g}, 2.36 \mathrm{mmol})$ as a colorless oil. I.R. (neat): 3291, 2955,

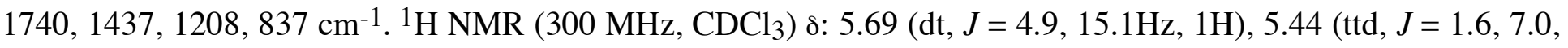
$15.1 \mathrm{~Hz}, 1 \mathrm{H}), 4.09(\mathrm{~d}, J=5.0 \mathrm{~Hz}, 2 \mathrm{H}), 3.72(\mathrm{~s}, 6 \mathrm{H}), 2.79(\mathrm{~d}, J=7.0 \mathrm{~Hz}, 2 \mathrm{H}), 2.77(\mathrm{~d}, J=2.5 \mathrm{~Hz}, 2 \mathrm{H}), 2.00(\mathrm{t}, J$ $=2.5 \mathrm{~Hz}, 1 \mathrm{H}), 0.88(\mathrm{~s}, 9 \mathrm{H}), 0.03(\mathrm{~s}, 6 \mathrm{H}) .{ }^{13} \mathrm{C} \mathrm{NMR}\left(75 \mathrm{MHz}, \mathrm{CDCl}_{3}\right) \delta: 170.1$ (2), 134.7, 122.9, 78.7, 71.4, 63.2, 56.9, 52.7 (2), 34.8, 25.8 (3), 22.5, 18.3, -5.2 (2). Anal. Calc'd for $\mathrm{C}_{9} \mathrm{H}_{13} \mathrm{NO}_{4}$ : C, 60.98; H, 8.52; Found: C, 60.79, H, 8.44.

\section{1-[[(E)-4-(tert-Butyldimethylsilyloxy)-but-2-enyl]-(4-methylbenzenesulfonyl)-amino]-prop-2-yne (8a)}

To a degassed solution of $\left(\eta^{3}-\mathrm{C}_{3} \mathrm{H}_{5} \mathrm{PdCl}\right)_{2}(0.094 \mathrm{~g}, 0.257 \mathrm{mmol})$, triphenylphosphine $(0.540 \mathrm{~g}, 2.05 \mathrm{mmol})$, cesium carbonate $(3.7 \mathrm{~g}, 11.39 \mathrm{mmol})$ and alkyne $7 \mathbf{a}(2.6 \mathrm{~g}, 12.4 \mathrm{mmol})$ in THF $(20 \mathrm{~mL})$, were added methyl carbonate 4 $(2.7 \mathrm{~g}, 10.3 \mathrm{mmol})$. The reaction mixture was heated at $60^{\circ} \mathrm{C}$ for $1 \mathrm{~h}$, and then partitioned between $\mathrm{Et}_{2} \mathrm{O}(100 \mathrm{~mL})$ and $1 \mathrm{~N} \mathrm{NaHSO}_{4}(100 \mathrm{~mL})$. The aqueous layer was extracted with $\mathrm{Et}_{2} \mathrm{O}(2 \times 100 \mathrm{~mL})$. The combined organic layers were dried over $\mathrm{MgSO}_{4}$, and concentrated in vacuo. The residue was chromatographed eluting with 6:1 petroleum ether : diethyl ether to afford $\mathbf{8 a}(3 \mathbf{3 7 g}, 8.56 \mathrm{mmol})$ as a colorless oil. I.R. (neat): 3275, 2929, 2119, 1598, 1350, $1163 \mathrm{~cm}^{-1} .{ }^{1} \mathrm{H}$ NMR $\left(500 \mathrm{MHz}, \mathrm{CDCl}_{3}\right): \delta 7.75(\mathrm{~d}, J=8.3 \mathrm{~Hz}, 2 \mathrm{H}), 7.31(\mathrm{~d}, J=8.3 \mathrm{~Hz}, 2 \mathrm{H}), 5.81(\mathrm{td}, J=4.4$, $15.1 \mathrm{~Hz}, 1 \mathrm{H}), 5.61(\mathrm{td}, J=6.8,15.1 \mathrm{~Hz}, 1 \mathrm{H}), 4.16(\mathrm{~d}, J=4.4 \mathrm{~Hz}, 2 \mathrm{H}), 4.10(\mathrm{~d}, J=2.4 \mathrm{~Hz}, 2 \mathrm{H}), 3.85(\mathrm{~d}, J=6.8 \mathrm{~Hz}$, $2 \mathrm{H}), 2.44(\mathrm{~s}, 3 \mathrm{H}), 2.01(\mathrm{t}, J=2.4 \mathrm{~Hz}, 1 \mathrm{H}), 0.91(\mathrm{~s}, 9 \mathrm{H}), 0.07(\mathrm{~s}, 6 \mathrm{H}) .{ }^{13} \mathrm{C}$ NMR $\left(125 \mathrm{MHz}, \mathrm{CDCl}_{3}\right): \delta 143.4,135.9$, 135.3, 129.4 (2), 127.7 (2), 122.9, 76.4, 73.6, 62.7, 47.8, 35.6, 25.8 (3), 21.5, 18.3, -5.2 (2). Anal. Calc'd for $\mathrm{C}_{20} \mathrm{H}_{31} \mathrm{NO}_{3} \mathrm{SSi}$ : C, 61.03; H, 7.94; N, 3.55; S, 8.14; Found: C, 60.77; H, 7.92; N, 3.42; S, 8.26.

\section{1-[[(E)-4-(tert-Butyldimethylsilyloxy)-but-2-enyl]-(4-methylbenzenesulfonyl)-amino]-but-3-yne (8b)}

To a degassed solution of $\left(\eta^{3}-\mathrm{C}_{3} \mathrm{H}_{5} \mathrm{PdCl}\right)_{2}(0.050 \mathrm{~g}, 0.137 \mathrm{mmol})$, triphenylphosphine $(0.300 \mathrm{~g}, 1.14 \mathrm{mmol})$, cesium carbonate $(2.3 \mathrm{~g}, 7.08 \mathrm{mmol})$ and alkyne $7 \mathbf{b}(1.7 \mathrm{~g}, 7.61 \mathrm{mmol})$ in THF $(20 \mathrm{~mL})$, were added methyl carbonate 4 $(1.6 \mathrm{~g}, 6.1 \mathrm{mmol})$. The reaction mixture was heated at $60^{\circ} \mathrm{C}$ for $1 \mathrm{~h}$, and then partitioned between $\mathrm{Et}_{2} \mathrm{O}(40 \mathrm{~mL})$ and $1 \mathrm{~N} \mathrm{NaHSO}_{4}(40 \mathrm{~mL})$. The aqueous layer was extracted with $\mathrm{Et}_{2} \mathrm{O}(2 \times 40 \mathrm{~mL})$. The combined organic layers were dried over $\mathrm{MgSO}_{4}$, and concentrated in vacuo. The residue was chromatographed eluting with 10:1 petroleum ether : diethyl ether to afford $\mathbf{8 b}(2.47 \mathrm{~g}, 6.05 \mathrm{mmol})$ as a colorless oil. I.R. (neat) : 3291, 2928, 2121, 1599, 1471, 1342, $1160 \mathrm{~cm}^{-1} .{ }^{1} \mathrm{H}$ NMR $\left(300 \mathrm{MHz}, \mathrm{CDCl}_{3}\right): \delta 7.68(\mathrm{~d}, J=8.0 \mathrm{~Hz}, 2 \mathrm{H}), 7.28(\mathrm{~d}, J=8.0 \mathrm{~Hz}, 2 \mathrm{H}), 5.68(\mathrm{td}, J=$ $4.1,15.4 \mathrm{~Hz}, 1 \mathrm{H}), 5.47(\mathrm{td}, J=7.8,15.4 \mathrm{~Hz}, 1 \mathrm{H}), 4.10(\mathrm{br} \mathrm{d}, J=1.7 \mathrm{~Hz}, 2 \mathrm{H}), 3.85(\mathrm{~d}, J=6.6 \mathrm{~Hz}, 1 \mathrm{H}), 3.27(\mathrm{t}, J=$ $7.3 \mathrm{~Hz}, 2 \mathrm{H}), 2.44(\mathrm{~m}, 2 \mathrm{H}), 2.43(\mathrm{~s}, 3 \mathrm{H}), 1.96(\mathrm{t}, J=1.7 \mathrm{~Hz}, 1 \mathrm{H}), 0.86(\mathrm{~s}, 9 \mathrm{H}), 0.02(\mathrm{~s}, 6 \mathrm{H}) .{ }^{13} \mathrm{C} \mathrm{NMR}(75 \mathrm{MHz}$ $\left.\mathrm{CDCl}_{3}\right): \delta 143.2,136.8,134.5,129.7$ (2), 127.0 (2), 123.8, 80.9, 70.0, 62.6, 50.0, 45.6, 25.8 (3), 21.4, 19.3, 18.2, - 
5.3 (2). Anal. Calc'd for $\mathrm{C}_{21} \mathrm{H}_{33} \mathrm{NO}_{3} \mathrm{SSi}$ : C, 61.87; H, 8.16; S, 7.86; N, 3.43; Found: C, 61.86; H, 7.96; N, 3.54; S, 7.96 .

\section{Methyl 4-[[(E)-4-(tert-Butyldimethylsilyloxy)-but-2-enyl]-(4-methylbenzenesulfonyl)-amino]-but-2-ynoate (9a)}

To a cold $\left(-78^{\circ} \mathrm{C}\right)$ solution of alkyne 8a $(1 \mathrm{~g}, 2.54 \mathrm{mmol})$ in THF $(12 \mathrm{ml})$ was added $n$-BuLi $(1.6 \mathrm{~N}, 1.8 \mathrm{ml}$, $2.88 \mathrm{mmol})$. The solution was stirred for $1 \mathrm{~h}$ at $-78^{\circ} \mathrm{C}$, and methyl chloroformate $(1 \mathrm{~mL})$ was added dropwise. The reaction was allowed to warm up over $1 \mathrm{~h}$, and water $(10 \mathrm{~mL})$ was added. The aqueous phase was extracted with $\mathrm{Et}_{2} \mathrm{O}(4 \mathrm{x} 20 \mathrm{~mL})$. The combined ethereal extracts were washed with brine $(20 \mathrm{~mL})$ and dried over $\mathrm{MgSO}_{4}$. The residue was chromatographed eluting with 6:1 petroleum ether : diethyl ether to afford 9a $(0.912 \mathrm{~g}, 2.02 \mathrm{mmol})$ as a colorless oil. I.R. (neat): 2955, 2240, 1719, 1352, 1255, 1164, $837 \mathrm{~cm}^{-1} .{ }^{1} \mathrm{H} \mathrm{NMR}\left(500 \mathrm{MHz}, \mathrm{CDCl}_{3}\right): \delta 7.74(\mathrm{~d}, J$ $=8.3 \mathrm{~Hz}, 2 \mathrm{H}), 7.33(\mathrm{~d}, J=8.3 \mathrm{~Hz}, 2 \mathrm{H}), 5.82(\mathrm{dt}, J=4.2,15.3 \mathrm{~Hz}, 1 \mathrm{H}), 5.62(\mathrm{dt}, J=6.9,15.1 \mathrm{~Hz}, 1 \mathrm{H}), 4.21(\mathrm{~s}, 2 \mathrm{H})$, $4.17(\mathrm{~d}, J=4.2 \mathrm{~Hz}, 2 \mathrm{H}), 3.83(\mathrm{~d}, J=6.9 \mathrm{~Hz}, 2 \mathrm{H}), 3.71(\mathrm{~s}, 3 \mathrm{H}), 2.44(\mathrm{~s}, 3 \mathrm{H}), 0,90(\mathrm{~s}, 9 \mathrm{H}), 0.6(\mathrm{~s}, 6 \mathrm{H}) .{ }^{13} \mathrm{C} \mathrm{NMR}$ (125 MHz, $\left.\mathrm{CDCl}_{3}\right): \delta 152.9,143.9,135.9,135.2,129.6$ (2), 127.6 (2), 122.3, 80.4, 76.8, 62.6, 52.6, 48.3, 35.5, 25.8 (3), 21.5, 18.3, -5.2 (2). Anal. Calc'd for $\mathrm{C}_{9} \mathrm{H}_{13} \mathrm{NO}_{4}$ : C, 58.50; H, 7.36; N, 3.10; Found: C, 58.38; H, 7.31; N, 3.06 .

Methyl 5-[[(E)-4-(tert-Butyldimethylsilyloxy)-but-2-enyl]-(4-methylbenzenesulfonyl)-amino]-pent-2-ynoate (9b) was obtained using the same protocol used for the preparation of 9a. The crude mixture was chromatographed eluting with $5: 1$ petroleum ether : diethyl ether to afford $\mathbf{9 b}$ in $75 \%$ yield as a colorless oil. I.R. (neat): 2954, 2242, 1716, 1435, 1341, $1257 \mathrm{~cm}^{-1} .{ }^{1} \mathrm{H}$ NMR (300 MHz, CDCl3): $\delta 7.68(\mathrm{~d}, J=8.3 \mathrm{~Hz}, 2 \mathrm{H}), 7.29(\mathrm{~d}, J=8.3 \mathrm{~Hz}$, $2 \mathrm{H}), 5.79(\mathrm{td}, J=4.4,15.1 \mathrm{~Hz}, 1 \mathrm{H}), 5.47(\mathrm{td}, J=6.6,15.1 \mathrm{~Hz}, 1 \mathrm{H}), 4.10(\mathrm{dd}, J=1.2,4.4 \mathrm{~Hz}, 2 \mathrm{H}), 3.84(\mathrm{~d}, J=$ $6.6 \mathrm{~Hz}, 2 \mathrm{H}), 3.28(\mathrm{t}, J=7.8 \mathrm{~Hz}, 2 \mathrm{H}), 2.61(\mathrm{t}, J=7.8 \mathrm{~Hz}, 2 \mathrm{H}), 2.41(\mathrm{~s}, 3 \mathrm{H}), 0.86(\mathrm{~s}, 9 \mathrm{H}), 0.01(\mathrm{~s}, 6 \mathrm{H}) .{ }^{13} \mathrm{C} \mathrm{NMR}(75$ $\mathrm{MHz}_{\mathrm{CDCl}}$ ): $\delta 153.7,143.5,136.4,134.9,129.7$ (2), 127.0 (2), 123.5, 85.8, 74.1, 62.5, 52.6, 50.3, 44.7, 25.8 (3), 21.4, 19.7, 18.2, -5.3 (2). Anal. Calc'd for $\mathrm{C}_{23} \mathrm{H}_{35} \mathrm{NO}_{5} \mathrm{SSi}$ : C, 59.32; H, 7.57; S, 6.88; N, 3.00; Found: C, 59.09; H, 7.67; N, 3.06; S, 6.67.

\section{1-[[(E)-4-(tert-Butyldimethylsilyloxy)-but-2-enyl]-(4-methylbenzenesulfonyl)-amino]-3-trimethylsilyl-prop-} 2-yne (10)

To a cold $\left(-78^{\circ} \mathrm{C}\right)$ solution of alkyne 8a $(0.904 \mathrm{~g}, 2.3 \mathrm{mmol})$ in THF $(12 \mathrm{~mL})$ was added $n$-BuLi $(1.6 \mathrm{~N}, 1.8 \mathrm{ml}$, $2.88 \mathrm{mmol})$. The solution was stirred for $20 \mathrm{~min}$. at $-78^{\circ} \mathrm{C}$, and trimethylsilyl chloride $(1 \mathrm{~mL})$ was added dropwise. The reaction was allowed to warm up over $1 \mathrm{~h}$, and water $(10 \mathrm{~mL})$ was added. The aqueous phase was extracted with $\mathrm{Et}_{2} \mathrm{O}(4 \mathrm{x} 20 \mathrm{~mL})$. The combined ethereal extracts were washed with brine $(20 \mathrm{~mL})$ and dried over $\mathrm{MgSO}_{4}$.

The residue was chromatographed eluting with $6: 1$ petroleum ether : diethyl ether to afford $\mathbf{1 0}(0.660 \mathrm{~g}, 1.41 \mathrm{mmol})$ as a colorless oil. I.R. (neat): 2956, 2178, 1646, 147, $1251 \mathrm{~cm}^{-1} .{ }^{1} \mathrm{H} \mathrm{NMR}\left(300 \mathrm{MHz}, \mathrm{CDCl}_{3}\right): \delta 7.71(\mathrm{~d}, J=$ $8.3 \mathrm{~Hz}, 2 \mathrm{H}), 7.27(\mathrm{~d}, J=8.3 \mathrm{~Hz}, 2 \mathrm{H}), 5.77(\mathrm{td}, J=4.6,15.3 \mathrm{~Hz}, 1 \mathrm{H}), 5.59(\mathrm{td}, J=6.6,15.9 \mathrm{~Hz}, 1 \mathrm{H}), 4.14(\mathrm{~m}, 2 \mathrm{H})$, $4.07(\mathrm{~s}, 2 \mathrm{H}), 3.80(\mathrm{~d}, J=6.5 \mathrm{~Hz}, 2 \mathrm{H}), 2.40(\mathrm{~s}, 3 \mathrm{H}), 0.88(\mathrm{~s}, 9 \mathrm{H}), 0.04(\mathrm{~s}, 6 \mathrm{H}),-0.02(\mathrm{~s}, 9 \mathrm{H}) .{ }^{13} \mathrm{C} \mathrm{NMR}(75 \mathrm{MHz}$, 
$\left.\mathrm{CDCl}_{3}\right): \delta 143.2,136.0,135.2,129.4(2), 127.7$ (2), 123.0, 97.7, 90.8, 62.8, 47.6, 36.6, 25.8 (3), 21.4, 18.3, -0.45 (3), -5.3 (2). Anal. Calc'd for $\mathrm{C}_{23} \mathrm{H}_{39} \mathrm{NO}_{\mathrm{S}} \mathrm{SSi}$ : C, 59.30; H, 8.44; N, 3.00; S, 6.88; Found: C, 59.12; H, 8.60; N, $2.91 ; \mathrm{S}, 7.12$.

\section{1-[[(Z)-4-(tert-Butyldimethylsilyloxy)-but-2-enyl]-(4-methylbenzenesulfonyl)-amino]-prop-2-yne (12 a)}

To an ice cooled solution of alcohol $11(0.430 \mathrm{~g}, 2.11 \mathrm{mmol})$ in THF $(12 \mathrm{~mL})$ was added triphenylphosphine $(0.666 \mathrm{~g}, 2.54 \mathrm{mmol})$, tosylamine $7 \mathbf{a}(0.532 \mathrm{~g}, 2.54 \mathrm{mmol})$ and diisopropyl azodicarboxylate $(0.5 \mathrm{~mL}, 2.54 \mathrm{mmol})$. The reaction mixture was stirred at room temperature for $2 \mathrm{~h}$. The volatiles were removed under vacuum. The residue was directly purified by chromatography eluting with 5:1 petroleum ether : diethyl ether to yield alkyne 12a $(0.648 \mathrm{~g}, 1.57 \mathrm{mmol})$ as a colorless oil. I.R. (neat): 3274, 2929, 1350, $1162 \mathrm{~cm}^{-1}$. ${ }^{1} \mathrm{H} \mathrm{NMR}(300 \mathrm{MHz}$, $\left.\mathrm{CDCl}_{3}\right): \delta 7.75(\mathrm{~d}, J=8.3 \mathrm{~Hz}, 2 \mathrm{H}), 7.31(\mathrm{~d}, J=8.3 \mathrm{~Hz}, 2 \mathrm{H}), 5.79(\mathrm{ddt}, J=1,5.8,11.2 \mathrm{~Hz}, 1 \mathrm{H}), 5.40(\mathrm{td}, J=7.3$, $11.2 \mathrm{~Hz}, 1 \mathrm{H}), 4.26(\mathrm{br} \mathrm{d}, J=5.8 \mathrm{~Hz}, 2 \mathrm{H}), 4.10(\mathrm{~d}, J=2.4 \mathrm{~Hz}, 2 \mathrm{H}), 3.90(\mathrm{~d}, J=7.3 \mathrm{~Hz}, 2 \mathrm{H}), 2.45(\mathrm{~s}, 3 \mathrm{H}), 2.02(\mathrm{t}, J=$ 2.4Hz, 1H), 0.89 (s, 9H), $\left.0.06(\mathrm{~s}, 6 \mathrm{H}) .{ }^{13} \mathrm{C} \mathrm{NMR} \mathrm{(125} \mathrm{MHz,} \mathrm{CDCl}_{3}\right): \delta$ 143.5, 135.8, 135.4, 129.4 (2), 127.7 (2), 123.8, 76.4, 73.6, 59.0, 43.2, 35.7, 25.8 (3), 21.5, 18.2, -5.2 (2). Anal. Calc'd for $\mathrm{C}_{20} \mathrm{H}_{31} \mathrm{NO}_{3} \mathrm{SSi}$ : C, 61.02; H, 7.93 ; N, 3.55; Found: C, 61.35; H, 7.52; N, 3.69.

\section{Methyl 4-[[(Z)-4-(tert-butyldimethylsilyloxy)-but-2-enyl]-(4-methylbenzenesulfonyl)-amino]-but-2-ynoate} (13)

To a cold $\left(-78^{\circ} \mathrm{C}\right)$ solution of alkyne $12 \mathrm{a}(0.340 \mathrm{~g}, 0.863 \mathrm{mmol})$ in THF $(5 \mathrm{~mL})$ was added $n$-BuLi $(1.6 \mathrm{~N}, 0.7 \mathrm{~mL}$, $1.12 \mathrm{mmol})$. The solution was stirred for $1 \mathrm{~h}$ at $-78^{\circ} \mathrm{C}$, and methyl chloroformate $(0.5 \mathrm{~mL})$ was added dropwise. The reaction was allowed to warm up over $1 \mathrm{~h}$, and water $(10 \mathrm{~mL})$ was added. The aqueous phase was extracted with $\mathrm{Et}_{2} \mathrm{O}(4 \mathrm{x} 20 \mathrm{~mL})$. The combined ethereal extracts were washed with brine $(20 \mathrm{~mL})$ and dried over $\mathrm{MgSO}_{4}$. The residue was chromatographed eluting with 5:1 petroleum ether : ether to give alkynoate $13(0.346 \mathrm{~g}, 0.766 \mathrm{mmol})$ as a colorless oil. I.R. (neat): 3029, 2955, 2240, 1720, 1256, $1164 \mathrm{~cm}^{-1}$.

${ }^{1} \mathrm{H}$ NMR $\left(500 \mathrm{MHz}, \mathrm{CDCl}_{3}\right) \delta: 7.73(\mathrm{~d}, J=8.3 \mathrm{~Hz}, 2 \mathrm{H}), 7.32(\mathrm{~d}, J=8.3 \mathrm{~Hz}, 2 \mathrm{H}), 5.80(\mathrm{ttd}, J=1.5,6.1,11 \mathrm{~Hz}, 1 \mathrm{H})$, $5.37(\mathrm{ttd}, J=1.5,7.3,11 \mathrm{~Hz}, 1 \mathrm{H}), 4.23(\mathrm{dd}, J=1.7,6.1 \mathrm{~Hz}, 2 \mathrm{H}), 4.20(\mathrm{~s}, 2 \mathrm{H}), 3.88(\mathrm{~d}, J=7.1 \mathrm{~Hz}, 2 \mathrm{H}), 3.69(\mathrm{~s}, 3 \mathrm{H})$, $2.43(\mathrm{~s}, 3 \mathrm{H}), 0.87(\mathrm{~s}, 9 \mathrm{H}), 0.04(\mathrm{~s}, 6 \mathrm{H}) .{ }^{13} \mathrm{C} \mathrm{NMR}\left(125 \mathrm{MHz}, \mathrm{CDCl}_{3}\right) \delta: 152.7,143.9,135.9,135.1,129.6(2)$, 127.5, 123.3, 80.4, 76.8, 58.8, 52.5, 43.6, 35.7, 25.7 (3), 21.4, 18.1, -5.4 (2).

HRMS : Calc'd for $\mathrm{C}_{21} \mathrm{H}_{30} \mathrm{NO}_{5} \mathrm{SSi}:\left[\mathrm{M}^{+}-\mathrm{CH}_{3}\right]$ 436.1613; Found : 436.1610.

\section{1-[[(Z)-4-(tert-Butyldimethylsilyloxy)-but-2-enyl]-(4-methylbenzenesulfonyl)-amino]-but-3-yne (12b)}

To an ice cooled solution of alcohol $11(0.86 \mathrm{~g}, 4.22 \mathrm{mmol})$ in THF $(25 \mathrm{~mL})$ was added triphenylphosphine $(1.33 \mathrm{~g}$, $5.07 \mathrm{mmol})$, tosylamine $\mathbf{7 b}(1.2 \mathrm{~g}, 5.0 \mathrm{mmol})$ and diisopropyl azodicarboxylate $(1.0 \mathrm{~mL}, 5.1 \mathrm{mmol})$. The reaction mixture was stirred at room temperature for $2 \mathrm{~h}$. The volatiles were removed under vacuum. The residue was directly purified by chromatography eluting with $3: 1$ petroleum ether : ether to yield alkyne $\mathbf{1 2 b}(1.005 \mathrm{~g}$, 2.46mmol) as a colorless oil. I.R. (neat): 3310, 2929, 1345, 1160, $1092 \mathrm{~cm}^{-1}$. ${ }^{1} \mathrm{H}$ NMR (500 MHz, $\left.\mathrm{CDCl}_{3}\right): \delta 7.72$ 
$(\mathrm{d}, J=8.3 \mathrm{~Hz}, 2 \mathrm{H}), 7.32(\mathrm{~d}, J=8.3 \mathrm{~Hz}, 2 \mathrm{H}), 5.69(\mathrm{ttd}, J=1.5,6.1,11.2 \mathrm{~Hz}, 1 \mathrm{H}), 5.33(\mathrm{ttd}, J=1.8,7.0,11.2 \mathrm{~Hz}, 1 \mathrm{H})$, $4.21(\mathrm{dd}, J=1.8,6.1 \mathrm{~Hz}, 1 \mathrm{H}), 3.96(\mathrm{~d}, J=7.0 \mathrm{~Hz}, 1 \mathrm{H}), 3.30(\mathrm{~d}, J=7.5 \mathrm{~Hz}, 1 \mathrm{H}), 3.29(\mathrm{~d}, J=7.5 \mathrm{~Hz}, 1 \mathrm{H}), 2.50(\mathrm{dd}, J$ $=2.7,7.8 \mathrm{~Hz}, 1 \mathrm{H}), 2.49(\mathrm{dd}, J=2.7,7.8 \mathrm{~Hz}, 1 \mathrm{H}), 2.45(\mathrm{~s}, 3 \mathrm{H}), 1.99(\mathrm{t}, J=2.7 \mathrm{~Hz}, 1 \mathrm{H}), 0.89(\mathrm{~s}, 9 \mathrm{H}), 0.07(\mathrm{~s}, 6 \mathrm{H})$. ${ }^{13} \mathrm{C}$ NMR (125 MHz, $\mathrm{CDCl}_{3}$ ): $\delta$ 143.3, 136.7, 133.6, 129.7 (2), 127.1 (2), 125.1, 80.9, 70.1, 59.1, 46.1, 45.4, 25.8 (3), 21.5, 19.5, 18.2, -5.2 (2). Anal. Calc'd for $\mathrm{C}_{21} \mathrm{H}_{33} \mathrm{NO}_{3} \mathrm{SSi}$ : C, 61.87; H, 8.15; S, 7.86; N, 3.43; Found: C, $61.79 ; \mathrm{H}, 8.33 ; \mathrm{S}, 7.97 ; \mathrm{N}, 3.66$.

\section{Ethyl (4S)-4-hydroxy-pent-2-enoate (16)}

To a solution of silyl ether $\mathbf{1 5}(4 \mathrm{~g})$, in $\mathrm{CH}_{2} \mathrm{Cl}_{2}(50 \mathrm{ml})$ was added water $(10 \mathrm{~mL})$ and trifluoroacetic acid $(20 \mathrm{ml})$. The reaction was stirred at room temperature for 15 min., and the volatiles were removed under reduced pressure. The residue was chromatographed eluting with $1: 1$ diethyl ether : petroleum ether to give $\alpha, \beta$-unsaturated ester 16 $(2.05 \mathrm{~g}, 14.2 \mathrm{mmol})$ as a clear oil. $[\alpha]_{\mathrm{D}}=23.4\left(\mathrm{c} 2.1, \mathrm{CHCl}_{3}\right)$. I.R. (neat): $3433,2980,1718,1657,1272 \mathrm{~cm}^{-1} .{ }^{1} \mathrm{H}$ $\operatorname{NMR}\left(500 \mathrm{MHz}, \mathrm{CDCl}_{3}\right) \delta: 6.97(\mathrm{dd}, J=4.7,15.6 \mathrm{~Hz}, 1 \mathrm{H}), 6.04(\mathrm{dd}, J=1.7,15.6 \mathrm{~Hz}, 1 \mathrm{H}), 4.51(\mathrm{ddq}, J=1.7,4.7$, $6.6 \mathrm{~Hz}, 1 \mathrm{H}), 4.22(\mathrm{q}, J=7.0 \mathrm{~Hz}, 2 \mathrm{H}), 1.88(\mathrm{~d}, 1 \mathrm{H}), 1.36(\mathrm{~d}, J=6.6 \mathrm{~Hz}, 2 \mathrm{H}), 1.31(\mathrm{t}, J=7.0 \mathrm{~Hz}, 3 \mathrm{H}) .{ }^{13} \mathrm{C} \mathrm{NMR}(125$ $\left.\mathrm{MHz}, \mathrm{CDCl}_{3}\right) \delta: 166.5,150.8,119.6,67.2,60.4,22.7,14.2$.

\section{Experimental procedure for preparation of tosylamines $17 \mathrm{a}$ and $17 \mathrm{~b}$}

Table 1

\begin{tabular}{|c|c|c|c|}
\hline $\begin{array}{c}\mathbf{1 6} \\
(\mathrm{g}, \mathrm{mmol})\end{array}$ & $\begin{array}{c}\text { Tosylamines } \\
(\mathrm{g}, \mathrm{mmol})\end{array}$ & $\begin{array}{c}\mathrm{PPh}_{3} \\
(\mathrm{~g}, \mathrm{mmol})\end{array}$ & $\begin{array}{c}\text { DIAD } \\
(\mathrm{ml}, \mathrm{mmol})\end{array}$ \\
\hline $0.5 ; 3.46$ & $\mathbf{7 a} ; 1.07,5.2$ & $1.35,5.14$ & $1,5.1$ \\
$0.576,4$ & $\mathbf{7 b} ; 1,4.47$ & $1.35,5.14$ & $1,5.1$ \\
\hline
\end{tabular}

To an ice cooled solution of alcohol 16 (see Table 1) in THF (0.2M) was added triphenylphosphine (see Table 1), tosylamine (see Table 1) and diisopropyl azodicarboxylate (see Table 1). The reaction mixture was stirred at room temperature for $2 \mathrm{~h}$. The volatiles were removed under vacuum. The residue was directly purified by chromatography eluting with $3: 2$ petroleum ether : diethyl ether $3: 2$ then $1: 1 \mathrm{v} / \mathrm{v}$ ) to yield impure alkyne (see Table 2) as a colorless oil.

\section{Experimental procedure for preparation of silyl ether 19a and 19b}




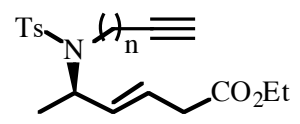

$17 \mathbf{a} \mathrm{n}=1$

$17 \mathrm{~b} \mathrm{n}=2$
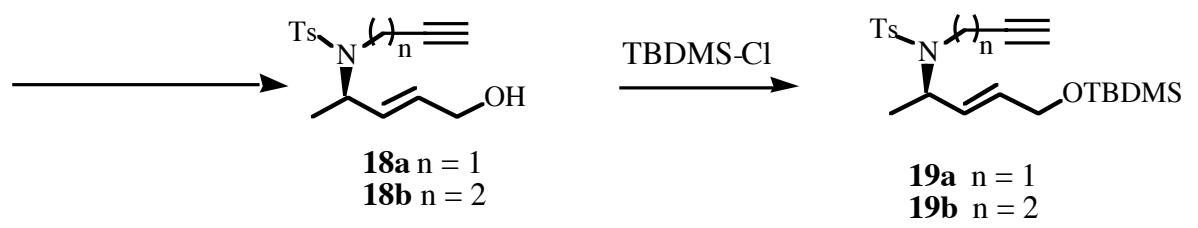

Table 2

\begin{tabular}{|c|c|c|c|c|c|}
\hline $\begin{array}{c}\text { Alkyne } \\
(\mathrm{g})\end{array}$ & $\begin{array}{c}\text { DIBAL-H } \\
(\mathrm{mL})\end{array}$ & $\begin{array}{c}\text { Alcohol } \\
(\mathrm{g}, \mathrm{mmol})\end{array}$ & $\begin{array}{c}\text { DMAP } \\
(\mathrm{g}, \mathrm{mmol})\end{array}$ & $\begin{array}{c}\text { TBDMS-Cl } \\
(\mathrm{g}, \mathrm{mmol})\end{array}$ & $\begin{array}{c}\text { Products } \\
(\mathrm{g}, \mathrm{mmol})\end{array}$ \\
\hline $\mathbf{1 7 a} ; 1$ & 8 & $\mathbf{1 8 a}, 0.5,1.7$ & $0.42,3.43$ & $0.26,1.7$ & $\mathbf{1 9 a} ; 0.592,1.45$ \\
$\mathbf{1 7 b}, 1.2$ & 10 & $\mathbf{1 8 b}, 0.74,2.4$ & $0.6,4.91$ & $0.36,2.4$ & $\mathbf{1 9 b} ; 0.898,2.12$ \\
\hline
\end{tabular}

To a cold $\left(-78^{\circ} \mathrm{C}\right.$ ) solution of impure alkyne (see Table 2) in $\mathrm{Et}_{2} \mathrm{O}(30 \mathrm{ml})$, was added DIBAL-H (1M, see Table 2). The reaction was stirred at $-78^{\circ} \mathrm{C}$ until completion (between 20 and $30 \mathrm{~min}$.). Water (one tenth of the DIBAL-H volume introduced) and $\mathrm{Et}_{2} \mathrm{O}(30 \mathrm{~mL})$ were added. The reaction mixture was stirred one hour at room temperature and the heterogeneous mixture was filtered over Celite $\left(\mathrm{Et}_{2} \mathrm{O}\right)$. The filtrate was evaporated to dryness. The residue was chromatographed (Pet.Eth. : $\mathrm{Et}_{2} \mathrm{O}$ 1:3 v/v) to yield the corresponding alcohol (see Table 2). The latter substance was taken up in $\mathrm{CH}_{2} \mathrm{Cl}_{2}(0.4 \mathrm{M})$. 4-DMAP (see Table 2), and $t$-butyldimethylsilyl chloride (see Table 2) were added. The reaction was stirred at room temperature until completion (around $1 \mathrm{~h}$ ). After concentration in vacuo, the residue was directly chromatographed eluting withh 9:1 petroleum ether : diethyl ether to afford the corresponding product (see Table 2) as a colorless oil.

\section{1-[[(4R)-(E)-4-(tert-Butyldimethylsilyloxy)-4-methyl-but-2-enyl]-(4-methylbenzenesulfonyl)-amino]-prop-2- yne (19a)}

$[\alpha]_{\mathrm{D}}=28.4$ (c 2.37, $\mathrm{CHCl}_{3}$ ). I.R. (neat): 3277, 2955, 1337, $1156 \mathrm{~cm}^{-1} .{ }^{1} \mathrm{H}$ NMR $\left(500 \mathrm{MHz}, \mathrm{CDCl}_{3}\right): \delta 7.81$ (d, $J$ $=8.3 \mathrm{~Hz}, 2 \mathrm{H}), 7.29(\mathrm{~d}, J=8.3 \mathrm{~Hz}, 2 \mathrm{H}), 5.66(\mathrm{dt}, 4.1,15.6 \mathrm{~Hz}, 1 \mathrm{H}), 5.61(\mathrm{dd}, J=4.4,15.6 \mathrm{~Hz}, 1 \mathrm{H}), 4.58(\mathrm{~m}, 1 \mathrm{H})$, $4.13(\mathrm{dd}, J=2.4,18.3 \mathrm{~Hz}, 1 \mathrm{H}), 4.13(\mathrm{~m}$ overlapped, $2 \mathrm{H}), 3.90(\mathrm{dd}, J=2.5,18.3 \mathrm{~Hz}, 1 \mathrm{H}), 2.43(\mathrm{~s}, 3 \mathrm{H}), 2.15(\mathrm{t}, J=$ $2.5 \mathrm{~Hz}, 1 \mathrm{H}), 1.31(\mathrm{~d}, J=6.8 \mathrm{~Hz}, 3 \mathrm{H}), 0.89(\mathrm{~s}, 9 \mathrm{H}), 0.05(\mathrm{~s}, 6 \mathrm{H}) .{ }^{13} \mathrm{C} \mathrm{NMR}\left(125 \mathrm{MHz}, \mathrm{CDCl}_{3}\right): \delta$ 143.1, 137.7 , 132.2, 129.3 (2), 128.4, 127.4 (2), 80.2, 72.2, 62.9, 54.1, 32.3, 25.8 (3), 21.5, 18.3, 17.8, -5.3 (2). Anal. Calc'd for $\mathrm{C}_{21} \mathrm{H}_{33} \mathrm{NO}_{3}$ SSi: C, 61.87; H, 8.15; S, 7.86; N, 3.43; Found: C, 61.70; H, 7.95; N, 3.45; S, 7.69.

\section{1-[[(4R)-(E)-4-(tert-Butyldimethylsilyloxy)-but-2-enyl]-(4-methylbenzenesulfonyl)-amino]-but-3-yne (19b)}

$[\alpha]_{\mathrm{D}}=50.7\left(\mathrm{c} 1.91, \mathrm{CHCl}_{3}\right)$. I.R. (neat) : 3310, 2929, 1341, $1154 \mathrm{~cm}^{-1} .{ }^{1} \mathrm{H}$ NMR $\left(500 \mathrm{MHz}, \mathrm{CDCl}_{3}\right): \delta 7.73(\mathrm{~d}, J$ $=8.3 \mathrm{~Hz}, 2 \mathrm{H}), 7.29(\mathrm{~d}, J=8.3 \mathrm{~Hz}, 2 \mathrm{H}), 5.60(\mathrm{ddt}, J=1.7,4.4,15.6 \mathrm{~Hz}, 1 \mathrm{H}), 5.45(\mathrm{ddt}, J=1,4.8,15.6 \mathrm{~Hz}, 1 \mathrm{H}), 4.54$ $(\mathrm{m}, 1 \mathrm{H}), 4.09(\mathrm{~m}, 1 \mathrm{H}), 3.26(\mathrm{ddd}, J=5.6,10.5,15.1 \mathrm{~Hz}, 1 \mathrm{H}), 3.18(\mathrm{ddd}, J=5.6,10.0,15.1 \mathrm{~Hz}, 1 \mathrm{H}), 2.64(\mathrm{dddd}, J=$ 2.7, 5.6, $10.2,15.9 \mathrm{~Hz}, 1 \mathrm{H}), 2.53(\mathrm{dddd}, J=2.4,5.6,0.2,15.9 \mathrm{~Hz}, 1 \mathrm{H}), 2.01(\mathrm{t}, J=2.4 \mathrm{~Hz}, 1 \mathrm{H}), 1.23(\mathrm{~d}, J=6.8 \mathrm{~Hz}$, 
3H), 0.88 (s, 9H), 0.03 (s, 6H). $\left.{ }^{13} \mathrm{C} \mathrm{NMR} \mathrm{(125} \mathrm{MHz,} \mathrm{CDCl}_{3}\right): \delta$ 143.1, 137.6, 132.0, 129.6 (2), 128.5, 127.1 (2), 81.1, 70.0, 62.8, 53.9, 42.5, 25.8 (3), 21.7, 21.4, 18.3, 18.1, -5.3 (2). Anal. Calc'd for $\mathrm{C}_{22} \mathrm{H}_{35} \mathrm{NO}_{3} \mathrm{SSi}$ : C, 62.66; H, 8.36; N, 3.32; S, 7.60; Found: C, 62.76; H, 8.26; N, 3.35; S, 7.67.

\section{Ethyl $(4 R, 5 S)$ 5-hydroxy-4-methyl-hex-2-enoate (21)}

To a solution of silyl ether $\mathbf{2 0}(4.0 \mathrm{~g}, 13.9 \mathrm{mmol})$ in $\mathrm{CH}_{2} \mathrm{Cl}_{2}(30 \mathrm{~mL})$ was added water $(5 \mathrm{~mL})$ and trifluoroacetic acid $(20 \mathrm{ml})$. The solution was stirred at room temperature for $15 \mathrm{~min}$. The volatiles were removed under reduced pressure and the residue was purified by chromatography eluting with $1: 1$ petroleum ether : diethyl ether to afford alcohol 21 (2.04g, 11.86mmol) as an oil. $[\alpha]_{\mathrm{D}}=29.7$ (c 1.17, $\mathrm{CH}_{2} \mathrm{Cl}_{2}$ ). I.R. (neat): 3444, 2976, $1718,1651 \mathrm{~cm}^{-1}$. ${ }^{1} \mathrm{H}$ NMR $\left(500 \mathrm{MHz}, \mathrm{CDCl}_{3}\right): \delta 6.94(\mathrm{dd}, J=8.3,15.6 \mathrm{~Hz}, 1 \mathrm{H}), 5.89(\mathrm{dd}, J=1.2,15.6 \mathrm{~Hz}, 1 \mathrm{H}), 4.20(\mathrm{q}, J=7.0 \mathrm{~Hz}$, 2H), 3.75 (app pent, $J=6.0 \mathrm{~Hz}, 1 \mathrm{H}), 2.36(\mathrm{~m}, 1 \mathrm{H}), 2.09(\mathrm{br} \mathrm{s}, 1 \mathrm{H}), 1.30(\mathrm{t}, J=7.0 \mathrm{~Hz}, 3 \mathrm{H}), 1.20(\mathrm{~d}, J=6.3 \mathrm{~Hz}, 3 \mathrm{H})$, $1.09(\mathrm{~d}, J=6.8 \mathrm{~Hz}, 3 \mathrm{H}) .{ }^{13} \mathrm{C} \mathrm{NMR}\left(125 \mathrm{MHz}, \mathrm{CDCl}_{3}\right): \delta 166.5,150.3,122.22,70.7,60.3,44.0,20.5,15.3,14.2$. Anal. Calc'd for $\mathrm{C}_{9} \mathrm{H}_{16} \mathrm{O}_{3}$ : C, 62.76; H, 9.36; Found: C, 62.54; H, 9.24.

\section{(2S,3R)6-(tert-Butyldimethylsilyloxy)-3-methyl-hex-4-en-2-ol (22)}

To a solution of alcohol $21(2.04 \mathrm{~g}, 11.4 \mathrm{mmol})$ in $\mathrm{CH}_{2} \mathrm{Cl}_{2}(25 \mathrm{~mL})$ were added successively pyridinium $p$ toluenesulfonate $(0.251 \mathrm{~g}, 1 \mathrm{mmol})$ and dihydropyrane $(2.7 \mathrm{~mL}, 30 \mathrm{mmol})$. The solution was stirred at room temperature for $8 \mathrm{~h}$. The solution was concentrated under reduced pressure and the residue directly chromatographed (P.Eth. : $\left.\mathrm{Et}_{2} \mathrm{O} 3: 1 \mathrm{v} / \mathrm{v}\right)$ to afford a colorless oil. The latter material was taken up in $\mathrm{Et}_{2} \mathrm{O}(60 \mathrm{ml})$. To the cold $\left(-78^{\circ} \mathrm{C}\right)$ solution was then added DIBAL-H $(1 \mathrm{M}, 30 \mathrm{~mL}, 30 \mathrm{mmol})$. The solution was stirred for $30 \mathrm{~min}$. at $-78^{\circ} \mathrm{C}$ and water $(3 \mathrm{~mL})$ and technical $\mathrm{Et}_{2} \mathrm{O}(30 \mathrm{~mL})$ were added. The solution was stirred or $1 \mathrm{~h}$ at room temperature. The heterogeneous solution was filtered over Celite $\left(\mathrm{Et}_{2} \mathrm{O}\right)$ and the filtrate concentrated in vacuo. The residue was purified by chromatography (petroleum ether : diethyl ether 1:2 v/v) to afford $2.1 \mathrm{~g}$ of a colorless oil. This material was dissolved in $\mathrm{CH}_{2} \mathrm{Cl}_{2}(25 \mathrm{ml})$. Triethylamine $(3.3 \mathrm{~mL}, 23.6 \mathrm{mmol})$, 4-DMAP $(0.250 \mathrm{~g}, 2.04 \mathrm{mmol})$ and $t$-butyldimethylsilyl chloride $(1.8 \mathrm{~g}, 12 \mathrm{mmol})$ were added. The solution was stirred at room temperature for $3 \mathrm{~h}$. Water $(20 \mathrm{~mL})$ was then added, and the aqueous phase was extracted with $\mathrm{CH}_{2} \mathrm{Cl}_{2}(3 \times 20 \mathrm{~mL})$. The combined organic layers were washed with brine $(30 \mathrm{~mL})$ and dried over $\mathrm{MgSO}_{4}$. After evaporation to dryness, the residue was purified by chromatography (petroleum ether : diethyl ether 10:1 v/v) to afford $2.936 \mathrm{~g}$ of a colorless oil.

To a solution of the latter $(2.8 \mathrm{~g}, 8.5 \mathrm{mmol})$ in $\mathrm{Et}_{2} \mathrm{O}(80 \mathrm{~mL})$ was added $\mathrm{MgBr}_{2} . \mathrm{OEt}_{2}(6.6 \mathrm{~g}, 25.5 \mathrm{mmol})$. The suspension was stirred at room temperature for $2 \mathrm{~h}$, and then was filtrated on a pad of silica gel (Et $2 \mathrm{O})$. To the filtrate was added water $(100 \mathrm{ml})$. The aqueous phase was extracted with $\mathrm{Et}_{2} \mathrm{O}(2 \times 50 \mathrm{~mL})$. The combined ethereal layers were dried over $\mathrm{MgSO}_{4}$ and concentrated in vacuo. The residue was chromatographed eluting with $2: 1$ petroleum ether : diethyl ether to afford first the remaining starting material $(0.7 \mathrm{~g})$ and then the alcohol $22(1.3 \mathrm{~g}$, $5.31 \mathrm{mmol})$ as a colorless oil. $[\alpha]_{\mathrm{D}}=15.6\left(\mathrm{c} 1.23, \mathrm{CHCl}_{3}\right)$. I.R. (neat) : 3419, 2958, 1255, $1095 \mathrm{~cm}^{-1} .{ }^{1} \mathrm{H} \mathrm{NMR}(500$ $\left.\mathrm{MHz} \mathrm{CDCl}_{3}\right) \delta: 5.63(\mathrm{td}, J=4.8,15.6 \mathrm{~Hz}, 1 \mathrm{H}), 5.52(\mathrm{dd}, J=7.8,15.6 \mathrm{~Hz}, 1 \mathrm{H}), 4.14(\mathrm{~d}, J=4.8 \mathrm{~Hz}, 2 \mathrm{H}), 3.53(\mathrm{pent}$, $J=6.4 \mathrm{~Hz}, 1 \mathrm{H}), 2.11(\mathrm{hex}, J=6.8 \mathrm{~Hz}, 1 \mathrm{H}), 1.13(\mathrm{~d}, J=6.4 \mathrm{~Hz}, 3 \mathrm{H}), 0.98(\mathrm{~d}, J=6.8 \mathrm{~Hz}, 3 \mathrm{H}), 0.88(\mathrm{~s}, 9 \mathrm{H}), 0.04(\mathrm{~s}$, 
6H). ${ }^{13} \mathrm{C} \mathrm{NMR}\left(125 \mathrm{MHz}, \mathrm{CDCl}_{3}\right) \delta: 132.3,131.3,70.9,63.6,44.4,25.8$ (3), 19.9, 18.3, 6.1, -5.1 (2). HRMS : Calc'd for $\mathrm{C}_{13} \mathrm{H}_{27} \mathrm{OSi}:\left[\mathrm{M}^{+}-\mathrm{OH}\right] 227.1831$; Found : 227.1842

\section{1-[[(2R,5R)-(E)-5-(tert-Butyldimethylsilyloxy)-1,2-dimethyl-pent-3-enyl]-(4-methylbenzenesulfonyl)-amino]- prop-2-yne (23)}

To an ice cooled solution of alcohol $22(0.63 \mathrm{~g}, 2.57 \mathrm{mmol})$ in THF $(13 \mathrm{~mL})$ was added triphenylphosphine $(1.35 \mathrm{~g}$, $5.15 \mathrm{mmol})$, tosylamine $7 \mathbf{a}(1.07 \mathrm{~g}, 5.15 \mathrm{mmol})$ and diisopropyl azodicarboxylate $(1.0 \mathrm{~mL}, 5.1 \mathrm{mmol})$. The reaction mixture was stirred at room temperature for $8 \mathrm{~h}$. The volatiles were removed under vacuum. The residue was directly purified by chromatography eluting with $3: 1$ petroleum ether : diethyl ether to yield alkyne $23(0.180 \mathrm{~g}$, $0.424 \mathrm{mmol})$ as a colorless oil. $[\alpha]_{\mathrm{D}}=5.2\left(\mathrm{c} 1.26, \mathrm{CHCl}_{3}\right)$. I.R. (neat) : 3310, 2930, 1340, $1156 \mathrm{~cm}^{-1} .{ }^{1} \mathrm{H} \mathrm{NMR}$ $\left(500 \mathrm{MHz} \mathrm{CDCl}_{3}\right) \delta: 7.76(\mathrm{~d}, J=8.3 \mathrm{~Hz}, 2 \mathrm{H}), 7.25(\mathrm{~d}, J=8.3 \mathrm{~Hz}, 2 \mathrm{H}), 5.57(\mathrm{td}, J=4.9,15.1 \mathrm{~Hz}, 1 \mathrm{H}), 5.42(\mathrm{dd}, J$ $=8.5,15.1 \mathrm{~Hz}, 1 \mathrm{H}), 4.10(\mathrm{~d}, J=4.9 \mathrm{~Hz}, 2 \mathrm{H}), 4.08(\mathrm{dd}$ partially overlapped, $J=2.4,18.5 \mathrm{~Hz}, 1 \mathrm{H}), 3.92(\mathrm{dd}, J=2.4$, $18.5 \mathrm{~Hz}, 1 \mathrm{H}), 3.67(\mathrm{dq}, J=6.8,9.5 \mathrm{~Hz}, 1 \mathrm{H}), 2.40(\mathrm{~s}, 3 \mathrm{H}), 2.36(\mathrm{~m}$ overlapped, $1 \mathrm{H}), 2.12(\mathrm{t}, J=2.4 \mathrm{~Hz}, 1 \mathrm{H}), 1.00(\mathrm{~d}$, $J=6.8 \mathrm{~Hz}, 3 \mathrm{H}), 0.98(\mathrm{~d}, J=6.8 \mathrm{~Hz}, 3 \mathrm{H}), 0.88(\mathrm{~s}, 9 \mathrm{H}), 0.04(\mathrm{~s}, 6 \mathrm{H}) .{ }^{13} \mathrm{C} \mathrm{NMR}\left(125 \mathrm{MHz}, \mathrm{CDCl}_{3}\right) \delta: 143.1,137.7$, 132.5, 130.5, 129.3 (2), 127.4 (2), 79.7, 72.3, 63.5, 58.3, 41.7, 31.9, 25.9 (2), 21.4, 18.6, 18.3, 16.6, -5.1 (2). HRMS : Calc'd for $\mathrm{C}_{19} \mathrm{H}_{28} \mathrm{NO}_{3} \mathrm{SSi}:\left[\mathrm{M}^{+}{ }_{-} \mathrm{C}_{4} \mathrm{H}_{9}\right] 378.1559$; Found : 378.1545.

\section{4,4-Dimethyl-9-trimethylsilyl-non-2-en-8-yn-1-ol}

To a solution of ester $\mathbf{2 8 b ^ { \mathrm { i } }}(3.9 \mathrm{~g}, 16.2 \mathrm{mmol})$ in $\mathrm{Et}_{2} \mathrm{O}(100 \mathrm{~mL})$ cooled at $-78^{\circ} \mathrm{C}$, was added DIBAL-H $(1 \mathrm{M}, 50 \mathrm{~mL}$, $50 \mathrm{mmol})$. The resulting solution was stirred for $20 \mathrm{~min}$. and water $(5 \mathrm{~mL})$ was added. The solution was stirred for $1 \mathrm{~h}$ at room temperature. The resulting mixture was filtered through Celite and thoroughly washed with Ether. The filtrate was then concentrated in vacuo to afford crude alcohol.To a solution oxalyl chloride $(2 \mathrm{~mL}, 22.9 \mathrm{mmol})$ in $\mathrm{CH}_{2} \mathrm{Cl}_{2}(50 \mathrm{~mL})$ was added at $-78^{\circ} \mathrm{C}$ a solution of DMSO $(3 \mathrm{~mL}, 42.2 \mathrm{mmol})$ in $\mathrm{CH}_{2} \mathrm{Cl}_{2}(10 \mathrm{~mL})$. After $10 \mathrm{~min}$., a solution of alcohol (crude, previously prepared), in $\mathrm{CH}_{2} \mathrm{Cl}_{2}(15 \mathrm{~mL})$ was added. The resulting mixture was stirred at $-78^{\circ} \mathrm{C}$ for $20 \mathrm{~min}$. and triethylamine $(10 \mathrm{~mL}, 70 \mathrm{mmol})$ was added slowly over $30 \mathrm{~min}$. The solution was then gradually warmed up to room temperature and stirred then for $1 \mathrm{~h}$. A saturated solution of $\mathrm{NaHCO}_{3}(30 \mathrm{~mL})$ was added. The aqueous layer was extracted with $\mathrm{CH}_{2} \mathrm{Cl}_{2}(3 \times 50 \mathrm{~mL})$. The combined organic layers were then dried over magnesium sulfate and concentrated in vacuo. The crude aldehyde was then dried azeotropically with toluene. The crude material was taken up in toluene $(100 \mathrm{~mL})$ and carboethoxymethylene triphenylphosphorane $(7 \mathrm{~g}$, $20 \mathrm{mmol}$ ) was added. The mixture was refluxed for $1 \mathrm{~h}$ and then evaporated to dryness. The residue was taken up in $3: 1$ petroleum ether : diethyl ether $(100 \mathrm{~mL})$. The suspension was then filtered over a pad of silica gel, and the pad thoroughly washed ( $3: 1$ petroleum ether : diethyl ether). The filtrate was then concentrated under reduced pressure, and the resulting residue $(\mathbf{2 8 b})$ was diluted with $\mathrm{Et} 2 \mathrm{O}(60 \mathrm{~mL})$. The solution was cooled to $-78^{\circ} \mathrm{C}$, and 
DIBAL-H (1M, 32mL, 32mmol) was added. The resulting solution was stirred for $20 \mathrm{~min}$. and water $(3.2 \mathrm{~mL})$ was added. The solution was stirred for $1 \mathrm{~h}$. at room temperature. The resulting mixture was filtered through Celite and thoroughly washed with $\mathrm{Et}_{2} \mathrm{O}$. The filtrate was then concentrated in vacuo. The residue was chromatographed eluting $1: 1$ petroleum ether : diethyl ether to afford allylic alcohol $(2.52 \mathrm{~g}, 11.0 \mathrm{mmol})$ as a colorless oil. I.R. (neat) : 3333, 2958, 2174, $1249 \mathrm{~cm}^{-1} .{ }^{1} \mathrm{H}$ NMR $\left(500 \mathrm{MHz}, \mathrm{CDCl}_{3}\right) \delta: 5.63(\mathrm{~d}, J=15.7 \mathrm{~Hz}, 1 \mathrm{H}), 5.55(\mathrm{td}, J=5.6,15.7 \mathrm{~Hz}$, $1 \mathrm{H}), 4.13(\mathrm{~d}, J=5.6 \mathrm{~Hz}, 2 \mathrm{H}), 2.18(\mathrm{t}, J=7.1 \mathrm{~Hz}, 2 \mathrm{H}), 1.45(\mathrm{~m}, 2 \mathrm{H}), 1.37(\mathrm{~m}, 2 \mathrm{H}), 1.01(\mathrm{~s}, 6 \mathrm{H}), 0.16(\mathrm{~s}, 9 \mathrm{H}) .{ }^{13} \mathrm{C}$ NMR (125 MHz, $\left.\mathrm{CDCl}_{3}\right): \delta 142.5,125.1,107.5,84.3,64.0,42.0,35.5,27.0,24.0,20.4,0.15$.

HRMS : Calc'd for $\mathrm{C}_{13} \mathrm{H}_{23} \mathrm{OSi}:\left[\mathrm{M}^{+}-\mathrm{CH}_{3}\right]$ 223.1518; Found : 223.1511.

\section{1-tert-Butyldimethylsilyloxy-4,4-dimethyl-non-2-en-8-yne (30b)}

To a solution of allylic alcohol $(0.750 \mathrm{~g}, 3.34 \mathrm{mmol})$ in $\mathrm{CH}_{2} \mathrm{Cl}_{2}(7 \mathrm{~mL})$ was added at room temperature 4-DMAP $(0.614 \mathrm{~g}, 5.0 \mathrm{mmol})$ and $t$-butyldimethylsilyl chloride $(0.510 \mathrm{~g}, 3.37 \mathrm{mmol})$. The solution was stirred for $1 \mathrm{~h}$. and then concentrated under reduced pressure. The residue was directly purified over silica gel eluting with $10: 1$ petroleum ether : diethyl ether to afford silyl ether $(1.04 \mathrm{~g})$. The latter material was dissolved in $\mathrm{MeOH}(14 \mathrm{~mL})$ and potassium carbonate $(0.4 \mathrm{~g})$ was added. The mixture was stirred overnight at room temperature. The volatiles were removed under reduced pressure, and the residue was filtered through a pad of silica gel eluting with $10: 1$ petroleum ether : diethyl ether to afford alkyne $30 \mathrm{~b}(0.760 \mathrm{~g}, 2.70 \mathrm{mmol})$ as a colorless oil. I.R. (neat): 3314, 2956, 2956, 2119, 1472, 1254, $1105 \mathrm{~cm}^{-1} .{ }^{1} \mathrm{H}$ NMR $\left(500 \mathrm{MHz}, \mathrm{CDCl}_{3}\right): \delta 5.57(\mathrm{~d}, J=15.6 \mathrm{~Hz}, 1 \mathrm{H}), 5.55(\mathrm{td}, J=5.4$, $15.6 \mathrm{~Hz}, 1 \mathrm{H}), 4.13(\mathrm{~d}, J=5.4 \mathrm{~Hz}, 2 \mathrm{H}), 2.18(\mathrm{td}, J=2.7,6.1 \mathrm{~Hz}, 2 \mathrm{H}), 1.95 \quad(\mathrm{t}, J=2.7 \mathrm{~Hz}, 11 \mathrm{H}), 1.46(\mathrm{~m}, 2 \mathrm{H})$, 1.39(m, 2H), $1.01(\mathrm{~s}, 6 \mathrm{H}), 0.92(\mathrm{~s}, 9 \mathrm{H}), 0.08(\mathrm{~s}, 6 \mathrm{H}) .{ }^{13} \mathrm{C} \mathrm{NMR}\left(125 \mathrm{MHz}, \mathrm{CDCl}_{3}\right): \delta$ 140.3, 125.5, 84.6, 68.1, 64.3, 42.0, 35.3, 27.0, 25.9, 23.8, 19.0, 18.4, -5.0. Calc'd for $\mathrm{C}_{19} \mathrm{H}_{28} \mathrm{NO}_{3} \mathrm{SSi}:\left[\mathrm{M}^{+}-\mathrm{C}_{4} \mathrm{H}_{9}\right]$ 223.1518; Found : 223.1502 .

\section{(E)-1-tert-Butyl-dimethylsilyloxy-4,4-dimethyl-oct-2-en-7-yne (30a)}

Starting from alkenoate 28a $(0.520 \mathrm{~g}, 2.0 \mathrm{mmol})$ and performing the same sequence, alkyne 30a $(0.334 \mathrm{~g}$, $1.25 \mathrm{mmol}$ ) was recovered as a colorless oil. I.R. (neat) : 3314, 2957, 2120, 1472, $1104 \mathrm{~cm}^{-1} .{ }^{1} \mathrm{H} \mathrm{NMR}(500 \mathrm{MHz}$, $\left.\mathrm{CDCl}_{3}\right) \delta: 5.2(\mathrm{~d}, J=15.7 \mathrm{~Hz}, 1 \mathrm{H}), 5.42(\mathrm{td}, J=4.6,15.7 \mathrm{~Hz}, 1 \mathrm{H}), 4.14(\mathrm{~d}, J=4.6 \mathrm{~Hz}, 2 \mathrm{H}), 2.07(\mathrm{~m}, 2 \mathrm{H}), 1.91(\mathrm{t}, J$ $=2.7 \mathrm{~Hz}, 1 \mathrm{H}), 1.57(\mathrm{~m}, 2 \mathrm{H}), 0.99(\mathrm{~s}, 6 \mathrm{H}), 0.90(\mathrm{~s}, 9 \mathrm{H}), 0.06(\mathrm{~s}, 6 \mathrm{H}) .{ }^{13} \mathrm{C} \mathrm{NMR}\left(125 \mathrm{MHz}, \mathrm{CDCl}_{3}\right) \delta: 139.1,126.3$, 85.2, 67.7, 64.1, 41.7, 35.5, 26.7 (2), 25.9 (3), 18.4, 14.0, -5.0 (2). Anal. Calc'd for $\mathrm{C}_{16} \mathrm{H}_{30} \mathrm{OSi}: \mathrm{C}, 72.11 ; \mathrm{H}$, 11.34; Found: C, 72.36; H, 11.30 .

\section{Methyl 9-(tert-butyldimethylsilyloxy)-6,6-dimethyl-non-7-en-2-ynoate 31}


To a cold $\left(-78^{\circ} \mathrm{C}\right)$ solution of alkyne $30 \mathrm{a}(0.230 \mathrm{~g}, 0.87 \mathrm{mmol})$ in THF $(12 \mathrm{ml})$ was added $n$-BuLi $(1.6 \mathrm{~N}, 0.7 \mathrm{ml}$, $1.12 \mathrm{mmol})$. The solution was stirred for $1 \mathrm{~h}$ at $-78^{\circ} \mathrm{C}$, and methyl chloroformate $(0.5 \mathrm{~mL})$ was added dropwise. The reaction was allowed to warm up over $1 \mathrm{~h}$, and water $(10 \mathrm{~mL})$ was added. The aqueous phase was extracted with $\mathrm{Et}_{2} \mathrm{O}$ ( 4 x 20mL). The combined ethereal extracts were washed with brine $(20 \mathrm{~mL})$ and dried over $\mathrm{MgSO}_{4}$. The residue was chromatographed eluting with $9: 1$ petroleum ether : diethyl ether to afford $\mathbf{3 1}(0.211 \mathrm{~g}, 0.65 \mathrm{mmol})$ as a colorless oil. I.R. (neat): 2956, 1718, 2239, $1254 \mathrm{~cm}^{-1} .{ }^{1} \mathrm{H} \mathrm{NMR}\left(500 \mathrm{MHz}, \mathrm{CDCl}_{3}\right) \delta: 5.52(\mathrm{~d}, J=15.6 \mathrm{~Hz}, 1 \mathrm{H})$, $5.47(\mathrm{td}, J=4.9,15.6 \mathrm{~Hz}, 1 \mathrm{H}), 4.18(\mathrm{~m}, 2 \mathrm{H}), 3.76(\mathrm{~s}, 3 \mathrm{H}), 2.23(\mathrm{dd}, J=8.1,8.3 \mathrm{~Hz}, 2 \mathrm{H}), 1.64(\mathrm{~m}, 2 \mathrm{H}), 1.01(\mathrm{~s}, 6 \mathrm{H})$, $0.92(\mathrm{~s}, 9 \mathrm{H}), 0.06(\mathrm{~s}, 6 \mathrm{H}) .{ }^{13} \mathrm{C} \mathrm{NMR}\left(125 \mathrm{MHz} \mathrm{CDCl}_{3}\right) \delta: 154.2,138.5,126.9,90.4,72.5,64.0,52.5,40.3,35.5$, 26.7 (2), 25.9 (3), 18.4, 14.4, -5.0 (2). Anal. Calc'd for $\mathrm{C}_{14} \mathrm{H}_{23} \mathrm{O}_{3} \mathrm{Si}\left[\mathrm{M}^{+}-\mathrm{C}_{4} \mathrm{H}_{9}\right]$ : 268.1494; Found: 268.1448

\section{(Z)-1-tert-Butyldimethylsilyloxy-4-(prop-2-ynyloxy)-but-2-ene 25}

To an ice cooled suspension of sodium hydride $(95 \%, 0.760 \mathrm{~g}, 30 \mathrm{mmol})$ in THF $(20 \mathrm{~mL})$ was added cis $1,4-$ but-2-ene-diol $(1.76 \mathrm{~g}, 20 \mathrm{mmol})$ in THF $(10 \mathrm{~mL})$. The suspension was stirred for $20 \mathrm{~min}$. at room temperature. The suspension was cooled down $\left(0^{\circ} \mathrm{C}\right)$ and tetrabutyl ammonium iodide $(0.1 \mathrm{~g}$, cat.) and propargyl bromide ( $80 \%$ in toluene, $2.4 \mathrm{~mL}, 22 \mathrm{mmol}$ ) were added. The solution was then stirred for $2 \mathrm{~h}$ at room temperature. Acetic acid $(2 \mathrm{~mL})$ was added and the volatiles were removed in vacuo. The residue was directly chromatographed (Pet.Eth. : Et $2 \mathrm{O}$ 9:1 then 2:1) to afford ether $24(0.730 \mathrm{~g}, 5.78 \mathrm{mmol})$.

The latter substance was taken up in $\mathrm{CH}_{2} \mathrm{Cl}_{2}(15 \mathrm{~mL})$. 4-DMAP $(1.06 \mathrm{~g}, 8.67 \mathrm{mmol})$ and $t$-butyldimethylsilyl chloride $(0.880 \mathrm{~g}, 5.85 \mathrm{mmol})$ were added. The reaction mixture was stirred for $2 \mathrm{~h}$ at room temperature. After evaporation to dryness, the residue was directly purified by chromatography eluting with $20: 1$ petroleum ether : diethyl ether to afford alkyne 25 (1.32g, $5.49 \mathrm{mmol})$. I.R. (neat): 3310, 2930, 1471, $1084 \mathrm{~cm}^{-1} .{ }^{1} \mathrm{H}$ $\operatorname{NMR}\left(500 \mathrm{MHz} \mathrm{CDCl}_{3}\right) \delta: 5.76(\mathrm{ttd}, J=1.4,5.8,11.2 \mathrm{~Hz}, 1 \mathrm{H}), 5.59(\mathrm{ttd}, J=1.7,6.6,11.2 \mathrm{~Hz}, 1 \mathrm{H}), 4.29$ $(\mathrm{dd}, J=1.7,5.8 \mathrm{~Hz}, 1 \mathrm{H}), 4.17$ (dd partially overlapped, $J=1.4,6.6 \mathrm{~Hz}, 1 \mathrm{H}), 4.16(\mathrm{~d}, J=2.5 \mathrm{~Hz}, 2 \mathrm{H}), 2.45(\mathrm{t}, J$ $=2.5 \mathrm{~Hz}, 1 \mathrm{H}), 0.92(\mathrm{~s}, 9 \mathrm{H}), 0.09(\mathrm{~s}, 6 \mathrm{H}) .{ }^{13} \mathrm{C} \mathrm{NMR}\left(125 \mathrm{MHz}, \mathrm{CDCl}_{3}\right) \delta: 133.7,125.8,79.6,74.4,65.1$, 59.4, 57.0, 25.9 (3), 18.3, -5.2 (2). Anal. Calc'd for $\mathrm{C}_{13} \mathrm{H}_{24} \mathrm{O}_{2} \mathrm{Si}$ : C, 64.94; H, 10.06; Found: C, 65.12; H, 9.96.

\section{Methyl (Z)-4-[4-(tert-butyldimethylsilyloxy)-but-2-enyloxy]-but-2-ynoate (26)}

To a cold $\left(-78^{\circ} \mathrm{C}\right)$ solution of alkyne $25(0.860 \mathrm{~g}, 3.55 \mathrm{mmol})$ in THF $(20 \mathrm{~mL})$ was added $n$-BuLi $(1.6 \mathrm{~N}, 2.4 \mathrm{~mL}$, $3.84 \mathrm{mmol})$. The solution was stirred for $1 \mathrm{~h}$ at $-78^{\circ} \mathrm{C}$, and methyl chloroformate $(0.6 \mathrm{ml})$ was added dropwise. The reaction was allowed to warm up over $1 \mathrm{~h}$, and water $(10 \mathrm{~mL})$ was added. The aqueous phase was extracted with $\mathrm{Et}_{2} \mathrm{O}(4 \times 20 \mathrm{~mL})$. The combined ethereal extracts were washed with brine $(20 \mathrm{~mL})$ and dried over $\mathrm{MgSO}_{4}$. The residue was chromatographed eluting with $9: 1$ petroleum ether : diethyl ether to afford first alkyne 25 (0.129g, $0.536 \mathrm{mmol})$ and then alkynoate $26(0.680 \mathrm{~g}, 2.27 \mathrm{mmol})$ as a colorless oil I.R. (neat) : 2955, 2238, 1722, 1254, 1087 $\mathrm{cm}^{-1} .{ }^{1} \mathrm{H}$ NMR $\left(500 \mathrm{MHz}, \mathrm{CDCl}_{3}\right) \delta: 5.77(\mathrm{ttd}, J=1.4,6.1,11.2 \mathrm{~Hz}, 1 \mathrm{H}), 5.57(\mathrm{ttd}, J=1.8,6.6,11.2 \mathrm{~Hz}, 1 \mathrm{H}), 4.28$ (s, 2H), 4.27 (tdd partially overlapped, $J=0.9,1.8,6.6 \mathrm{~Hz}, 1 \mathrm{H}), 4.17$ (tdd, $J=0.7,1.4,6.1 \mathrm{~Hz}, 1 \mathrm{H}), 3.8(\mathrm{~s}, 3 \mathrm{H}), 0.91$ 
(s, 9H), 0.09 (s, 6H). $\left.{ }^{13} \mathrm{C} \mathrm{NMR} \mathrm{(125} \mathrm{MHz,} \mathrm{CDCl}_{3}\right) \delta: 153.4,134.2,125.2,83.5,77.7,65.6,59.4,56.7,52.7,25.8$

(3), 18.2, -5.2 (2). Anal. Calc'd for $\mathrm{C}_{15} \mathrm{H}_{26} \mathrm{O}_{4} \mathrm{Si}$ : C, 60.36; H, 8.78; Found: C, 60.22; H, 8.60

\section{(1S))4-[[(E)-4-(tert-Butyldimethylsilyloxy)-1-methyl-but-2-enyl]-(4-methylbenzenesulfonyl)-amino]-but-2-}

\section{ynoic acid methyl ester 51}

To a cold $\left(-78^{\circ} \mathrm{C}\right)$ solution of alkyne 19a $(0.30 \mathrm{~g}, 0.70 \mathrm{mmol})$ in THF $(5 \mathrm{~mL})$ was added $n$-BuLi $(1.6 \mathrm{~N}, 0.6 \mathrm{~mL}, 0.9 \mathrm{c}$ mmol). The solution was stirred for $1 \mathrm{~h}$ at $-78^{\circ} \mathrm{C}$, and methyl chloroformate $(0.5 \mathrm{~mL})$ was added dropwise. Th reaction was allowed to warm up over $1 \mathrm{~h}$, and water $(10 \mathrm{~mL})$ was added. The aqueous phase was extracted with $\mathrm{Et}_{2} \mathrm{C}$ ( 4 x 20mL). The combined ethereal extracts were washed with brine $(20 \mathrm{~mL})$ and dried over $\mathrm{MgSO}_{4}$. Afte evaporattion to dryness, the residue was chromatographed eluting with $3: 1$ petroleum ether : diethyl ether $3: 1 \mathrm{t}$ give alkynoate $\mathbf{5 1}(0.282 \mathrm{~g}, 0.60 \mathrm{mmol})$ as a colorless oil. $[\alpha]_{\mathrm{D}}=18.4\left(\mathrm{c} 1.21, \mathrm{CHCl}_{3}\right)$. I.R. (neat): 2954, 2241,1719 1256, $1156 \mathrm{~cm}^{-1}$. ${ }^{1} \mathrm{H}$ NMR (500 MHz, $\left.\mathrm{CDCl}_{3}\right): \delta 7.79$ (d, J=8.3Hz, 2H), 7.29 (d, J = 8.3Hz, $\left.2 \mathrm{H}\right), 5.64(\mathrm{ttd}, 1.5$ $4.4,15.6 \mathrm{~Hz}, 1 \mathrm{H}), 5.53(\mathrm{ddt}, J=1.5,4.4,15.6 \mathrm{~Hz}, 1 \mathrm{H}), 4.55(\mathrm{~m}, 1 \mathrm{H}), 4.24(\mathrm{~d}, J=19.0 \mathrm{~Hz}, 1 \mathrm{H}), 4.11(\mathrm{td}, J=1.4$ $4.4 \mathrm{~Hz}, 2 \mathrm{H}), 3.98(\mathrm{~d}, J=19.0 \mathrm{~Hz}, 1 \mathrm{H}), 3.74(\mathrm{~s}, 3 \mathrm{H}), 2.41(\mathrm{~s}, 3 \mathrm{H}), 1.24(\mathrm{~d}, J=7.1 \mathrm{~Hz}, 3 \mathrm{H}), 0.86(\mathrm{~s}, 9 \mathrm{H}), 0.22(\mathrm{~s}, 6 \mathrm{H})$ ${ }^{13} \mathrm{C}$ NMR (125 MHz, $\left.\mathrm{CDCl}_{3}\right) \delta$ : 153.3, 143.5, 137.3, 132.8, 129.6 (2), 127.9, 127.4 (2), 84.0, 75.5, 62.8, 53.8, 52.6 32.1, 25.8 (3), 21.5, 18.3, 17.5, -5.3 (2). Anal. Calc'd for $\mathrm{C}_{23} \mathrm{H}_{35} \mathrm{NO}_{5} \mathrm{SSi}$ : C, 59.32; H, 7.57; N, 3.06; S, 6.88; Found C, 59.47; H, 7.36; N, 3.07; S, 6.69. HRMS : Calc'd for $\mathrm{C}_{23} \mathrm{H}_{35} \mathrm{NO}_{5} \mathrm{SSi}_{[}\left[\mathrm{M}^{+}\right]$465.2005; Found : 465.1997

N-(4-Methylbenzenesulfonyl)-34-(2-hydroxyethyl]-4-methoxycarbonyl-methylenepyrrolidine (67) To solution of silyl enol ether $34(0.120 \mathrm{~g}, 0.265 \mathrm{mmol})$ in $\mathrm{CH}_{2} \mathrm{Cl}_{2}(5 \mathrm{~mL})$ was added water $(0.1 \mathrm{~mL})$ and trifluoroaceti acid $(2 \mathrm{~mL})$. The reaction was stirred at room temperature for $15 \mathrm{~min}$. and the volatiles were removed under reducer pressure. The residue was purified by chromatography (eluting with 1:3 diethyl ether petroleum ether to afford th corresponding aldehyde $(0.088 \mathrm{~g}, 0.260 \mathrm{mmol})$ as a clear oil. I.R. (neat): 2846, 1188, 1658, $1164 \mathrm{~cm}^{-1} .{ }^{1} \mathrm{H} \mathrm{NMF}$ $\left(500 \mathrm{MHz}_{\mathrm{CDCl}}\right): \delta 9.74(\mathrm{~s}, 1 \mathrm{H}), 7.72(\mathrm{~d}, J=8.3 \mathrm{~Hz}, 2 \mathrm{H}), 7.34(\mathrm{~d}, J=8.3 \mathrm{~Hz}, 2 \mathrm{H}), 5.70(\mathrm{~s}, 1 \mathrm{H}), 5.30(\mathrm{~d}, J=$ $18.3 \mathrm{~Hz}, 1 \mathrm{H}), 4.21(\mathrm{dd}, J=2.4,18.3 \mathrm{~Hz}, 1 \mathrm{H}), 3.69(\mathrm{~s}, 3 \mathrm{H}), 3.48(\mathrm{dd}, J=7.4,9.5 \mathrm{~Hz}), 1 \mathrm{H}), 3.27(\mathrm{~m}, 1 \mathrm{H}), 2.94(\mathrm{~m}, 1 \mathrm{H})$ $2.75(\mathrm{dd}, J=4.6,18.8 \mathrm{~Hz}, 1 \mathrm{H}), 2.71(\mathrm{dd}, J=7.8,18.8 \mathrm{~Hz}, 1 \mathrm{H}), 2.43(\mathrm{~s}, 3 \mathrm{H}) .{ }^{13} \mathrm{C} \mathrm{NMR}\left(125 \mathrm{MHz}, \mathrm{CDCl}_{3}\right): \delta 198.7$ $165.7,160.2,143.9,131.9,129.7$ (2), 127.8 (2), 113.3, 52.1, 51.8, 51.4, 46.6, 38.0, 21.4. To a solution of thi aldehyde $(0.072 \mathrm{~g}, 0.213 \mathrm{mmol})$ in $\mathrm{MeOH}(5 \mathrm{~mL})$ was added $\mathrm{NaBH}_{4}(0.016 \mathrm{~g}, 0.426 \mathrm{mmol})$. The solution was stirrer at room temperature for $30 \mathrm{~min}$. and water $(2 \mathrm{~mL})$ was added. The mixture was then concentrated in vacuo. Th residue was diluted with water $(3 \mathrm{~mL})$ and extracted with $\mathrm{CH}_{2} \mathrm{Cl}_{2}(3 \times 10 \mathrm{~mL})$. The combined organic layers wer dried over $\mathrm{MgSO}_{4}$ and concentrated under reduced pressure. The residue was chromatographed eluting with 9: diethyl ether : petroleum ether to afford alcohol $67(0.065 \mathrm{~g}, 0.191 \mathrm{mmol})$ as a colorless oil. I.R. (film): 3540,2951 1713, 1667, 1345, $1162 \mathrm{~cm}^{-1} .{ }^{1} \mathrm{H}$ NMR $\left(300 \mathrm{MHz} \mathrm{CDCl}_{3}\right) \delta: 7.74(\mathrm{~d}, J=8.3 \mathrm{~Hz}, 2 \mathrm{H}), 7.34(\mathrm{~d}, J=8.3 \mathrm{~Hz}, 2 \mathrm{H}), 5,7$. (br d, $J=2.4 \mathrm{~Hz}, 1 \mathrm{H}), 4.30(\mathrm{dd}, J=2.7,18.3 \mathrm{~Hz}, 1 \mathrm{H}), 4.20(\mathrm{dt}, J=2.5,18.3 \mathrm{~Hz}, 1 \mathrm{H}), 3.69(\mathrm{~m}, 5 \mathrm{H}), 3.50(\mathrm{~m}, 1 \mathrm{H})$ 
$2.98(\mathrm{~m}, 2 \mathrm{H}), 2.43(\mathrm{~s}, 3 \mathrm{H}), 2.00(\mathrm{~s}, 1 \mathrm{H}), 1.86(\mathrm{~m}, 1 \mathrm{H}), 1.62(\mathrm{tq}, J=5.6,8.8 \mathrm{~Hz}, 1 \mathrm{H}) .{ }^{13} \mathrm{C} \mathrm{NMR}\left(75 \mathrm{MHz}, \mathrm{CDCl}_{3}\right) \delta$ 166.1, 161.6, 143.9, 131.7, 129.7 (2), 127.8 (2), 112.7, 60.0, 52.3, 51.9, 51.3, 41.4, 34.8, 21.4. Anal. Calc'd fo $\mathrm{C}_{16} \mathrm{H}_{21} \mathrm{NO}_{5} \mathrm{~S}: \mathrm{C}, 56.62 ; \mathrm{H}, 6.24 ; \mathrm{N}, 4.13 ; \mathrm{S}, 9.45$; Found: C, 56.91; H, 6.14; N, 4.17; S, 9.49.

\section{Methyl-[5-(4-methylbenzenesulfonyl)-hexahydro-furo[2,3-c]pyrrol-6a-yl]-acetate (68)}

I.R. (neat) : 2953, 1736, 1345, $1165 \mathrm{~cm}^{-1} .{ }^{1} \mathrm{H}$ NMR $\left(500 \mathrm{MHz}, \mathrm{CDCl}_{3}\right): \delta 7.65(\mathrm{~d}, J=8.3 \mathrm{~Hz}, 2 \mathrm{H}), 7.31(\mathrm{~d}, J=$ $8.3 \mathrm{~Hz}, 2 \mathrm{H}), 3.82(\mathrm{~d}, J=5.2 \mathrm{~Hz}, 1 \mathrm{H}), 3.81(\mathrm{~d}, J=5.4 \mathrm{~Hz}, 1 \mathrm{H}), 3.60(\mathrm{~s}, 3 \mathrm{H}), 3.43(\mathrm{~d}, J=10.8 \mathrm{~Hz}, 1 \mathrm{H}), 3.18(\mathrm{dd}, J=$ $7.9,9.8 \mathrm{~Hz}, 1 \mathrm{H}), 3.10(\mathrm{~d}, J=10.8 \mathrm{~Hz}, 1 \mathrm{H}), 3.05(\mathrm{dd}, J=3.8,9.8 \mathrm{~Hz}, 1 \mathrm{H}), 2.68$ (hept, $J=3.9 \mathrm{~Hz}, 1 \mathrm{H}), 2.58(\mathrm{~d}, J=$ $15.2 \mathrm{~Hz}, 1 \mathrm{H}), 2.54(\mathrm{~d}, J=15.2 \mathrm{~Hz}, 1 \mathrm{H}), 2.41(\mathrm{~s}, 3 \mathrm{H}), 2.10(\mathrm{qd}, J=8.1,11.5 \mathrm{~Hz}, 1 \mathrm{H}), 1.75(\mathrm{~m}, 1 \mathrm{H}) .{ }^{13} \mathrm{C} \mathrm{NMR}(12$. $\mathrm{MHz}_{\mathrm{CDCl}}$ ): $\delta$ 170.3, 143.7, 131.7, 129.6 (2), 128.0 (2), 89.5, 68.3, 58.2, 53.5, 51.7, 46.6, 41.8, 32.5, 21.5. Anal

Calc'd for $\mathrm{C}_{16} \mathrm{H}_{21} \mathrm{NO}_{5} \mathrm{~S}: \mathrm{C}, 56.62 ; \mathrm{H}, 6.24 ; \mathrm{N}, 4.13$; S, 9.45; Found: C, 56.84; H, 6.32; N, 4.27; S, 9.33.

\section{N-(4-Methylbenzenesulfonyl)-3-[2-(2-Oxo-dihydro-furan-3-ylidene)-ethyl]-4-methoxy-}

carbonylmethylenepyrrolidine (69). To a solution of aldehyde $(0.083 \mathrm{~g}, 0.246 \mathrm{mmol})$ in $\mathrm{CH}_{2} \mathrm{Cl}_{2}(3 \mathrm{ml})$ was adder 2-triphenyphosphoranylide- $\gamma$-butyrolactone $(0.170 \mathrm{~g}, 0.492 \mathrm{mmol})$. The reaction mixture was stirred at roon temperature for $90 \mathrm{~min}$. The crude mixture was concentrated in vacuo and the residue was purified on silica ge eluting with $4: 1$ ethyl acetate : petroleum ether to afford lactone $69(0.092 \mathrm{~g}, 0.227 \mathrm{mmol})$ as a white solid. m.p.: 91 $92^{\circ}$ C. I.R. (neat) : 2951, 1754, 1714, 1670, 1436, 1346, $1163 \mathrm{~cm}^{-1} .{ }^{1} \mathrm{H} \mathrm{NMR}\left(500 \mathrm{MHz}, \mathrm{CDCl}_{3}\right): \delta 7.69(\mathrm{~d}, J=$ $8.3 \mathrm{~Hz}, 2 \mathrm{H}), 7.33(\mathrm{~d}, J=8.3 \mathrm{~Hz}, 2 \mathrm{H}), 6.58(\mathrm{~m}, 1 \mathrm{H}), 5.75(\mathrm{br} \mathrm{s}, 1 \mathrm{H}), 4.39(\mathrm{~m}, 3 \mathrm{H}), 4.11(\mathrm{dd}, J=2.5,8.1 \mathrm{~Hz}, 1 \mathrm{H}), 3.6 i$ $(\mathrm{s}, 3 \mathrm{H}), 3.18(\mathrm{dd}, J=6.6,9.5 \mathrm{~Hz}, 1 \mathrm{H}), 3.04(\mathrm{dd}, J=4.9,9.5 \mathrm{~Hz}, 1 \mathrm{H}), 2.92(\mathrm{~m}, 2 \mathrm{H}), 2.82(\mathrm{~m}, 1 \mathrm{H}), 2.41(\mathrm{~s}, 3 \mathrm{H}), 2.4($ (m partially overlapped, $2 \mathrm{H}) .{ }^{13} \mathrm{C}$ NMR $\left(125 \mathrm{MHz} \mathrm{CDCl}_{3}\right): \delta 170.5,165.7,1599.8,144.0,135.2,131.6,129.8(2)$ 128.6, 127.8 (2), 113.6, 65.5, 52.2, 51.4, 51.1, 43.4, 33.2, 25.1, 21.4.

HRMS: Calc'd for $\mathrm{C}_{19} \mathrm{H}_{20} \mathrm{NO}_{5} \mathrm{~S}$ [M+- $\left.\mathrm{MH}_{3} \mathrm{O}\right]$ : 374.1062; Found: 374.1024.

\section{References}

1. Maier, M.E.; Schöffling, B. Chem. Ber. 1989, 122, 1081 